\title{
Robust Nonlinear State Feedback Under Structured Uncertainty
}

This work follows the global input/output linearization approach for the design of control systems for nonlinear plants of Kravaris and Chung. A robust nonlinear state feedback is proposed for uncertainties considered as a class of bounded perturbations to the state model. A Liapunov-based approach is used to guarantee uniform ultimate boundedness.

\author{
Costas Kravaris, Srinivas Palanki \\ Department of Chemical Engineering \\ University of Michigan \\ Ann Arbor, Ml 48109
}

\section{Introduction}

For all but the simplest control schemes to be effective, some description of the process to be controlled must be available. Usually this description is a mathematical model. However a series of difficulties may be encountered while developing a meaningful and realistic mathematical description of the chemical process. Thus for most processes, a "reasonable model" with some "good" values for the model parameters is employed for control purposes. The mismatch between the mathematical model and the true process can lead to serious stability problems for the process, especially when the process is nonlinear. Thus the design of robust control strategies that take care of model uncertainty is of paramount importance for the design of good and efficient control systems for a chemical process.

One of the most important contributions in control theory in the past decade has been the development of robust linear controller design methodologies for linear plants in the presence of unstructured uncertainty (Doyle and Stein, 1981). This approach can be applied to the control of nonlinear plants as well, with the understanding that the uncertainty will contain the error introduced by the linear approximation. The key issue is whether the linear approximation error will be small or not. In the case of mild nonlinearities, the uncertainty introduced by the linearization approximation is small enough so that it can successfully be rejected by a robust linear controller without too much sacrifice in performance. In the case of a highly nonlinear process, the frequency domain bounds corresponding to the linear approximation error will be very loose; they will contain not only the nonlinear uncertainties but also a very large class of unstructured uncertainties that fall within these bounds. This will lead to conservativism and therefore poor performance. Thus robust linear control theory is insufficient for highly non-

Correspondence concerning this paper should be addressed to Costas Kravaris. linear processes. A nonlinear control theory that provides robust controllers is needed.

For nonlinear state feedback synthesis under the assumption of a perfect model, geometric methods that provide closed-loop linearity characteristics have received considerable attention (Su, 1983; Hunt et al., 1983a, b; Kravaris and Chung, 1987; Gilbert and $\mathrm{Ha}, 1984$; Ha and Gilbert, 1987) and lead to relatively simple control laws. We use this framework to address the following robust design problem:

Given upper bounds on the modeling error of the nonlinear process, design a nonlinear state feedback law that guarantees stability and performance for all perturbations within the given bounds.

Our work considers the case of structured model uncertainty. The uncertainty need not be parametric but must be representable as a class of perturbations to the state model to which upper bounds (in general state-dependent) are available. At this point, our theory does not include unmodeled higher order dynamics.

The robust design problem is approached in two steps:

1. Under the assumption of a perfect model, do a nominal design of the state feedback (to meet the appropriate closed-loop linearity requirements and minimize some design criterion)

2. Introduce robustness corrections in accordance with the size of the modeling error

Throughout this paper we will follow the input/output linearization approach (Kravaris and Chung, 1987) to obtain the nominal design. We will first provide the necessary background (previous work and notation), and a brief survey of input/output linearizing feedback. Then we will present our robust design methodology. The application of the proposed method is illustrated in two examples. The Appendix provides a brief outline for computing Liapunov functions numerically. This may be useful in applying the proposed methodology. 


\section{Previous Work}

Nonlinear state feedback synthesis by means of differential geometric methods has received considerable attention in the literature. In all methods, linearity of the closed-loop system is sought in some sense: for example, linearity of the closed-loop state equations (Su, 1982; Hunt et al., 1983a, b) or linearity of the input/output map (Kravaris and Chung), or linearity of both state equations and input/output map (Gilbert and $\mathrm{Ha}$, 1984; Ha and Gilbert, 1987) or linearity and decoupling at the same time (Isidori et al., 1981). Once the necessary state feedback is computed, the natural question that arises is how robust it is with respect to modeling errors.

The use of Liapunov-based methods for robust controller design in conjunction with differential geometric methods was first introduced by $\mathrm{Ha}$ and Gilbert (1987). Their work addressed the case where the nominal state feedback provides linearity of both the state equations and the output. In this work we are going to solve the more general problem, where the nominal state feedback linearizes the closed-loop system only in an input/output sense. Both our work and that of $\mathrm{Ha}$ and Gilbert, (1987) can be viewed as generalizations of work pioneered by Gutman (1979), Leitmann (1981), and Corless and Leitmann (1981) and later explored by many others.

\section{Standard Differential-Geometric Notation}

Given $f$, a $C^{\infty}$ vector field on $R^{n}$, and $h$, a $C^{\infty}$ scalar field on $R^{n}$, the Lie derivative of $h$ with respect to $f$ is defined as

$$
L_{f}(h)=\langle d h, f\rangle
$$

where $\langle.,$.$\rangle denotes the inner product in R^{n}$ i.e.,

$$
\langle d h, f\rangle=\frac{\partial h}{\partial x_{1}} f_{1}+\cdots+\frac{\partial h}{\partial x_{n}} f_{n}
$$

This Lie derivative is also a $C^{\infty}$ scalar field on $R^{n}$. Thus, one can inductively define higher order Lie derivatives as follows:

$$
L_{f}^{k}(h)=L_{f}\left(L_{f}^{k-1}(h)\right)=\left\langle d L_{f}^{k-1}(h), f\right\rangle, k=2,3 \ldots
$$

Given $f, g C^{\infty}$ vector fields on $R^{n}$, the Lie bracket $[f, g]$ is a vector field defined by

$$
[f, g]=\frac{\partial g}{\partial x} f-\frac{\partial f}{\partial x} g
$$

where $\partial f / \partial x$ and $\partial g / \partial x$ are the Jacobians. $[f, g]$ is also a $C^{\infty}$ vector field on $R^{n}$. One can define successive Lie brackets $[f,[f, g]],[f,[f,[f, g]]]$, etc. We will use the standard notation

$$
\begin{aligned}
a d_{f}^{0}(g) & =g \\
a d_{f}^{1}(g) & =[f, g] \\
a d_{f}^{2}(g) & =[f,[f, g]] \\
& \vdots \\
\operatorname{adf}_{f}^{k}(g) & =\left[f, a d_{f}^{k-1}(g)\right]
\end{aligned}
$$

\section{Previous Results on Input/Output Linearization}

We restrict ourselves to single-input/single-output (SISO) nonlinear systems of the form

$$
\begin{aligned}
& \dot{x}=f(x)+g(x) u \\
& y=h(x)
\end{aligned}
$$

where $x \in R^{n}, u \in R, y \in R$, and to nonlinear static state feedback

$$
u=\Psi(x, v)
$$

Consider now Eq. 1 and the problem of finding a static state feedback of the form of Eq. 2 such that the $v-y$ input/output system is linear and of minimal order. This problem was posed and solved by Kravaris and Chung (1987). The results are summarized in the following theorem:

Theorem 1 (Kravaris and Chung, 1987). The minimal order of the $v-y$ system is the relative order of Eq. 1, i.e., the smallest integer $r$ satisfying

$$
\left\langle d h, a d_{f}^{r-1}(g)\right\rangle \neq 0
$$

The necessary state feedback is given by

$$
u=\frac{v-\sum_{k=0}^{r} \beta_{k} L_{f}^{k}(h)}{(-1)^{r-1} \beta_{r}\left\langle d h, a d_{f}^{r-1}(g)\right\rangle}
$$

where $\beta_{k}$ are arbitrarily selected numbers. The corresponding closed-loop response is given by

$$
\sum_{k=0}^{r} \beta_{k} \frac{d^{k} y}{d t^{k}}=v
$$

\section{Main Results}

We will consider here the problem of designing a robust controller so that the process output $y(t)$ will track a given set point $y_{s p}$. By introducing appropriate deviation variables we can rewrite our system so that $y$ represents the tracking error, the problem therefore being to control $y$ to zero.

Consider the model, Eq. 1, with the nominal state feedback, Eq. 3, where $v=0$. Also consider the "true" system

$$
\begin{aligned}
& \dot{x}=f^{\prime}(x)+g^{\prime}(x) u, \quad x(0)=x_{0} \\
& y=h^{t}(x)
\end{aligned}
$$

We would like to design a robustness correction

$$
v=\mathscr{R}(x)
$$

so that Eq. 5 with the state feedback, Eqs. 3 and 6, has tracking error that is uniformly ultimately bounded in the sense of the following definition:

Definition 1. The output of a dynamic system $y(t):[0, \infty] \rightarrow$ $R$ is uniformly ultimately bounded with ultimate bound $b>0$ if for every $r>0$ there exists $\tau(b, r)>0$ such that $\left\|x_{0}\right\| \leq r$ implies $|y(t)| \leq b$ for all $t \geq \tau$. 
In particular, we will design $\mathscr{R}$ so that the control system is robust in the following sense. Given suitable limitations on the modeling errors

$$
\Delta f=f^{\prime}-f, \Delta g=g^{\prime}-g, \Delta h=h^{t}-h
$$

the tracking error is uniformly ultimately bounded, where $b$ and $\tau$ are independent of the allowed $\Delta f, \Delta g$, and $\Delta h$.

Remark 1. $\tau$ can be interpreted as the settling time and therefore as a measure of performance.

Before stating the main theorem we state a few assumptions.

Assumption $A l . f, g$, and $h$ are smooth. $f^{t}, g^{t}$, and $h^{t}$ are $C^{0}$. $\Delta f \in \Sigma_{f}, \Delta g \in \Sigma_{g}, \Delta h \in \Sigma_{h}$ where $\Sigma_{f}, \Sigma_{g}$, and $\Sigma_{h}$ are specified sets.

Assumption $A 2$. The origin $x=0$ is a uniformly asymptotically stable equilibrium point of the unforced nominal system

$$
\dot{x}=f(x)-\frac{\sum_{k=0}^{r} \beta_{k} L_{f}^{k}(h)(x)}{(-1)^{r-1} \beta_{r}<d h, \operatorname{ad}_{f}^{r-1}(g)>(x)} g(x)
$$

In particular, there exists a $C^{\prime}$ function $V: R^{n} \rightarrow R^{+}$and continuous strictly increasing functions $\gamma_{i}: R^{+} \rightarrow R^{+}, i=1,2,3$ satisfying

$$
\begin{gathered}
\gamma_{i}(0)=0, \quad i=1,2,3 \\
\lim _{r \rightarrow \infty} \gamma_{i}(r)=\infty, \quad i=1,2
\end{gathered}
$$

such that

$$
\begin{gathered}
\gamma_{1}(\|x\|) \leq V(x) \leq \gamma_{2}(\|x\|) \\
\left|d V, f-\frac{\sum_{k=0}^{r} \beta_{k} L_{f}^{k}(h) g}{(-1)^{r-1} \beta_{r}\left(d h, a d_{f}^{r-1}(g)\right\rangle}\right| \leq-\gamma_{3}(\|x\|)
\end{gathered}
$$

In other words, there is a Liapunov function $V$ for the unforced nominal system.

Assumption A3. $h(x)$ vanishes at the origin $x=0$.

Assumption $A 4$. For each $\Delta f \epsilon \Sigma_{f}$ and $\Delta g \epsilon \Sigma_{g}$ there exist mappings $\Delta f^{*}: R^{n} \rightarrow R$ and $\Delta g^{*}: R^{n} \rightarrow R$ satisfying

$$
(-1)^{r-1}\left\langle d h, a d_{f}^{r-1}(g)\right\rangle \Delta f=g \Delta f^{*}, \quad \Delta g=g \Delta g^{*}
$$

for all $x \in R^{n}$. that

Assumption A5. There exists a $C^{0}$ function $\mu: R^{n} \rightarrow R$ such

$$
1+\Delta g^{*} \geq \mu(x)>0
$$

for every $\Delta g \in \Sigma_{g}$. Moreover, $\mu(x)$ is bounded:

$$
\mu^{0} \geq \mu(x) \quad \forall x \in R^{n}
$$

Assumption $A 6$. There exists a $C^{0}$ function $\phi: R^{n} \rightarrow R$ such that

$$
\phi(x) \geq \frac{1}{\mu(x)}\left|\Delta f^{*}-\left[\sum_{k=0}^{r} \beta_{k} L_{f}^{k}(h)\right] \Delta g^{*}\right|
$$

for every $\Delta f \in \Sigma_{f}$ and $\Delta g \in \Sigma_{g}$.
Assumption A7. There exists $\delta_{h}>0$ such that

$$
|\Delta h| \leq \delta_{h}
$$

for every $\Delta h \in \Sigma_{h}$.

Assumption Al is a technical assumption for mathematical completeness. Assumption A2 tells us that the nominal closedloop system must be internally stable in the sense that there exists a Liapunov function. Note that this is a very natural assumption; unless the nominal system is internally stable, we cannot expect robustness properties. Assumption A3 implies that $y$ represents the tracking error. A given system can easily be rewritten in this form by suitably defining appropriate deviation variables. Assumption A4 defines the matching conditions of our theory. The uncertainties $\Delta f$ and $\Delta g$ have the structure defined by assumption A4. This condition restricts the class of perturbations for which the method is applicable. Note however that this condition is much weaker than the standard conicity assumption for $\Delta f$ of the small gain theorem method (Safonov, 1980; Kantor, 1987) and the standard assumption of vanishing $\Delta$ g. Assumptions A5-A7 define the uncertainty bands (in general state-dependent) for $\Delta f, \Delta g$, and $\Delta h$. The following Theorem provides an explicit formula for the robustness correction (Eq. 17), which guarantees uniform ultimate boundedness of the output. For a proof, the reader is referred to Kravaris and Palanki (1988).

Theorem 2. Assume that $\mathbf{A} 1-\mathbf{A} 7$ are satisfied. Consider

$$
\begin{aligned}
& \dot{x}=[f(x)+\Delta f]+[g(x)+\Delta g] u, \quad x(0)=x_{0} \\
& y=h(x)+\Delta h
\end{aligned}
$$

where $\Delta f \in \Sigma_{g}, \Delta g \in \Sigma_{g}, \Delta h \in \Sigma_{h}$, and the state feedback

$$
u=\frac{v-\sum_{k=0}^{r} \beta_{k} L_{f}^{k}(h)}{(-1)^{r-1} \beta_{r}\left\langle d h, a d_{f}^{r-1}(g)\right\rangle}
$$

where

$$
v=-\phi(x) \eta_{\epsilon}\left[\frac{\mu^{0} \phi(x)\langle d V, g\rangle(x)}{(-1)^{r-1} \beta_{r}\left(d h, a d_{f}^{r-1}(g)\right\rangle(x)}\right]
$$

and where $\eta_{\epsilon}$ is the saturation function

$$
\eta_{\epsilon}(\zeta)= \begin{cases}\frac{\zeta}{\epsilon}, & \text { if }|\zeta| \leq \epsilon \\ \frac{\zeta}{|\zeta|} & \text { if }|\zeta|>\epsilon\end{cases}
$$

with

$$
\epsilon<\lim _{r \rightarrow \infty} \gamma_{3}(r)
$$

Then for every $\Delta f \in \Sigma_{f}, \Delta g \in \Sigma_{g}$ and $\Delta h \in \Sigma_{h}$ the output is uniformly ultimately bounded. More precisely, for every $b>$ $\left(\gamma_{1}^{-1} \circ \gamma_{2} \circ \gamma_{3}^{-1}\right)(\epsilon / 4)$, we have

$$
|y(t)| \leq\|h\|_{L i p} b+\delta_{h} \quad \forall t \geq \tau\left(b,\left\|x_{0}\right\|\right)
$$




$$
\tau\left(b,\left\|x_{0}\right\|\right)= \begin{cases}0, & \text { if }\left\|x_{0}\right\| \leq\left(\gamma_{2}^{-1} \circ \gamma_{1}\right)(b) \\ \frac{\gamma_{2}\left(\left\|x_{0}\right\|\right)-\gamma_{1} \circ \gamma_{2}^{-1} \circ \gamma_{1}(b)}{\gamma_{3} \circ \gamma_{2}^{-1} \circ \gamma_{1}(b)-\frac{\epsilon}{4}} & \text { if }\left\|x_{0}\right\|>\left(\gamma_{2}^{-1} \circ \gamma_{1}\right)(b)\end{cases}
$$

and $\|h\|_{\text {Lip }}$ is the Lipschitz norm of $h$ in $\left(x \in R^{n}:\|x\| \leq b\right\}$.

Remark 2. Equation 17 is a continuous approximation (as $\epsilon \rightarrow 0$ ) of the discontinuous control law

$$
v=-\phi(x) \operatorname{sgn}\left[\frac{\langle d V, g\rangle(x)}{(-1)^{r-1} \beta_{r}\left\langle d h, a d_{f}^{r-1}(h)\right\rangle(x)}\right]
$$

which, of course, cannot be implemented in practice (due to its discontinuity).

Remark 3. From Eq. 17 we observe that we are feeding back the uncertainty band $\phi(x)$ with a nonlinear gain. The theory indicates that we should choose as small an $\epsilon$ as possible. However too small an $\epsilon$ may excite unmodeled higher order dynamics, which are not taken into account in the theory. In practice one will need to choose $\epsilon$ either by simulation experiments or fine-tune $\epsilon$ on-line.

\section{Illustrative Examples}

\section{Example 1}

Consider a continuously stirred tank reactor (CSTR) in which an isothermal, liquid-phase, multicomponent chemical reaction is being carried out. The chemical reaction system is:

$$
A=B \rightarrow C
$$

with the rates of reaction given by:

$$
\begin{aligned}
& r_{1}=k_{1} C_{A}-k_{2} C_{B}^{2} \\
& r_{2}=k_{3} C_{B}^{2}
\end{aligned}
$$

There is an unmodeled first-order side reaction from $B$. Also there is an error in the flow-rate/valve-position relationship; this creates an error in measuring the molar feed rate of $B$. It is desired to control $C_{C}$ as close as possible to the steady state value by adjusting the molar feed rate of $B$, Figure 1 .

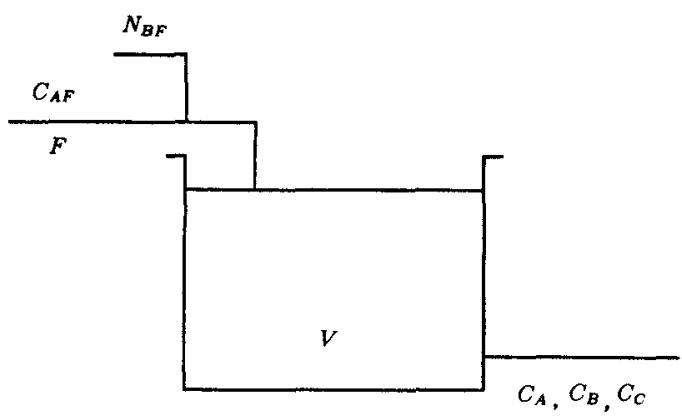

Figure 1. System for example 1.
The modeling equations for this system in terms of dimensionless variables are (see the Notation for explanation of symbols):

$$
\begin{aligned}
\frac{d x_{1}}{d t} & =1-x_{1}-D a_{1} x_{1}+D a_{2} x_{2}^{2} \\
\frac{d x_{2}}{d t} & =D a_{1} x_{1}-x_{2}-D a_{2} x_{2}^{2}-D a_{3} x_{2}^{2}+u \\
\frac{d x_{3}}{d t} & =D a_{3} x_{2}^{2}-x_{3} \\
y & =x_{3}
\end{aligned}
$$

where $u=N_{B F} / F C_{A F}$.

The steady state values $x_{1 D}, x_{2 D}, x_{3 D}, u_{D}$ are defined by

$$
\begin{aligned}
& 0=1-x_{1 D}-D a_{1} x_{1 D}+D a_{2} x_{2 D}^{2} \\
& 0=D a_{1} x_{1 D}-x_{2 D}-D a_{2} x_{2 D}^{2}-D a_{3} x_{2 D}^{2}+u_{D} \\
& 0=D a_{3} x_{2 D}^{2}-x_{3 D}
\end{aligned}
$$

Defining the deviation variables $\bar{x}_{1}=x_{1}-x_{1 D}, \bar{x}_{2}=x_{2}-x_{2 D}$, $\bar{x}_{3}=x_{3}-x_{30}, \bar{u}=u-u_{D}$, the model becomes:

$$
\begin{aligned}
\frac{d \bar{x}_{1}}{d t}= & -\left(1+D a_{1}\right) \bar{x}_{1}+\left(2 D a_{2} x_{2 D}\right) \bar{x}_{2}+\left(D a_{2}\right) \bar{x}_{2}^{2} \\
\frac{d \bar{x}_{2}}{d t}= & D a_{1} \bar{x}_{1}-\left(1+2 D a_{2} x_{2 D}+2 D a_{3} x_{2 D}\right) \bar{x}_{2} \\
& -\left(D a_{2}+D a_{3}\right) \bar{x}_{2}^{2}+\bar{u} \\
\frac{d \bar{x}_{3}}{d t}= & D a_{3} \bar{x}_{2}^{2}+\left(2 D a_{3} x_{2 D}\right) \bar{x}_{2}-\bar{x}_{3} \\
\bar{y}= & \bar{x}_{3}
\end{aligned}
$$

Thus we have:

True system

$$
\begin{aligned}
& \dot{x}=[f(x)+\Delta f]+[g(x)+\Delta g] \bar{u} \\
& y=h(x)+\Delta h
\end{aligned}
$$

Modeled system

$$
\begin{aligned}
& \dot{x}=f(x)+g(x) \bar{u} \\
& y=h(x)
\end{aligned}
$$




$$
f(x)=\left[\begin{array}{c}
-\left(1+D a_{1}\right) \bar{x}_{1}+\left(2 D a_{2} x_{2 D}\right) \bar{x}_{2}+\left(D a_{2}\right) \bar{x}_{2}^{2} \\
D a_{1} \bar{x}_{1}-\left(1+2 D a_{2} x_{2 D}+2 D a_{3} x_{2 D}\right) \bar{x}_{2}-\left(D a_{2}+D a_{3}\right) \bar{x}_{2}^{2} \\
D a_{3} \bar{x}_{2}^{2}+\left(2 D a_{3} x_{2 D}\right) \bar{x}_{2}-\bar{x}_{3}
\end{array}\right]
$$$$
g(x)=\left[\begin{array}{l}
0 \\
1 \\
0
\end{array}\right], h(x)=\bar{x}_{3}
$$$$
\Delta f=\left[\begin{array}{c}
0 \\
-e_{f 1} \bar{x}_{2} \\
0
\end{array}\right] \text { and } \Delta g=\left[\begin{array}{c}
0 \\
\epsilon_{g 1} \\
0
\end{array}\right]
$$

The values of the various constants are as follows:

$$
\begin{aligned}
& D a_{1}=3.0 \quad D a_{2}=0.5 \quad D a_{3}=1.0 \\
& e_{f 1}=0.05 \quad \epsilon_{g_{1}}=0.3 \quad u_{D}=1.0
\end{aligned}
$$

The steady state values of the states are:

$$
x_{1 D}=0.3467 \quad x_{2 D}=0.8796 \quad x_{3 D}=0.8796
$$

To Develop a Robust Controller Based on the Modeled System.

1. Calculation of relative order.

It can be easily verified that $\langle d h, g\rangle=0$ and $\left\langle d h, a d_{f}^{1}(g)\right\rangle \neq$ 0 and thus the relative order of the system is 2 .

2. Calculation of state feedback control law.

From Eq. 16,

$$
\bar{u}=\frac{v-\left\{\beta_{0} \bar{x}_{3}+\beta_{1}\left(D a_{3} \bar{x}_{2}^{2}+2 D a_{3} x_{2 D} \bar{x}_{2}-\bar{x}_{3}\right)+\beta_{2}\left[\left(2 D a_{3} \bar{x}_{2}+2 D a_{3} x_{2 D}\right) f_{2}-f_{3}\right]\right\}}{\beta_{2}\left(2 D a_{3} \bar{x}_{2}+2 D a_{3} x_{2 D}\right)}
$$

where

$$
\begin{aligned}
& f_{2}=D a_{1} \bar{x}_{1}-\left(1+2 D a_{2} x_{2 D}+2 D a_{3} x_{2 D}\right) \bar{x}_{2}-\left(D a_{2}+D a_{3}\right) \bar{x}_{2}^{2} \\
& f_{3}=D a_{3} \bar{x}_{2}^{2}+\left(2 D a_{3} x_{2 D}\right) \bar{x}_{2}-\bar{x}_{3}
\end{aligned}
$$

Making this substitution in the modeled system we have

$$
\begin{aligned}
\frac{d \bar{x}_{1}}{d t}= & -\left(1+D a_{1}\right) \bar{x}_{1}+\left(2 D a_{2} x_{2 D}\right) \bar{x}_{2}+\left(D a_{2} \bar{x}_{2}^{2}\right) \\
\frac{d \bar{x}_{2}}{d t}= & \frac{v}{\beta_{2}\left(2 D a_{3} \bar{x}_{2}+2 D a_{3} x_{2 D}\right)}-\frac{\beta_{0} \bar{x}_{3}}{\beta_{2}\left(2 D a_{3} \bar{x}_{2}+2 D a_{3} x_{2 D}\right)} \\
& -\left(\frac{\beta_{1}}{\beta_{2}}-1\right) \frac{\left[D a_{3} \bar{x}_{2}^{2}+\left(2 D a_{3} x_{2 D}\right) \bar{x}_{2}-\bar{x}_{3}\right]}{\beta_{2}\left(2 D a_{3} \bar{x}_{2}+2 D a_{3} x_{2 D}\right)} \\
\frac{d \bar{x}_{3}}{d t}= & D a_{3} \bar{x}_{2}^{2}+\left(2 D a_{3} x_{2 D}\right) \bar{x}_{2}-\bar{x}_{3} \\
\bar{y}= & \bar{x}_{3}
\end{aligned}
$$

We obtain the "unforced system" by putting $v=0$. It is obvious that the origin is an equilibrium point of the unforced system.

Pick $\beta_{0}=1.0, \beta_{1}=1.0, \beta_{2}=1.0$.

3. Lyapunov function for the unforced system.

It can easily be verified that the following positive definite function:

$$
\begin{aligned}
V=84 \bar{x}_{1}^{2}+ & 681\left(\bar{x}_{2}^{2}+1.7592 \bar{x}_{2}-\bar{x}_{3}\right)^{2}+1,012 \bar{x}_{3}^{2} \\
& +20 \bar{x}_{1}\left(\bar{x}_{2}^{2}+1.7592 \bar{x}_{2}-\bar{x}_{3}\right)+16 \bar{x}_{1} \bar{x}_{3} \\
& +680 \bar{x}_{3}\left(\bar{x}_{2}^{2}+1.7592 \bar{x}_{2}-\bar{x}_{3}\right)
\end{aligned}
$$

has the time derivative:

$$
\dot{V}=-672\left[\bar{x}_{1}^{2}+\left(\bar{x}_{2}^{2}+1.7592 \bar{x}_{2}-\bar{x}_{3}\right)^{2}+\bar{x}_{3}^{2}\right]
$$

which is negative definite. Thus $V$ is a desirable Lyapunov func. tion.

4. Calculation of $\Delta f^{*}, \Delta g^{*}(\mathrm{Eq} .10)$ :

$$
\begin{aligned}
& \Delta f^{*}=-0.05 \bar{x}_{2}\left(2 \bar{x}_{2}+1.7592\right) \\
& \Delta g^{*}=0.3
\end{aligned}
$$

5. Calculation of $\mu(x), \mu^{0}$ (Eq. 12):

$$
\begin{aligned}
1+\Delta g^{*} & \geq \mu(x) \geq 0 \\
\mu^{0} & \geq \mu(x)
\end{aligned}
$$

As $\Delta g^{*}=0.3$ set $\mu(x)=\mu^{0}=0.3$.

6. Calculation of $\phi(x)$ (Eq. 13). Set:

$$
\begin{aligned}
\phi(x)=\frac{1}{0.3} \mid-0.05 \bar{x}_{2}\left(2 \bar{x}_{2}+1.7592\right)-0.3 \\
\cdot\left[3 \bar{x}_{1}+\left(2 \bar{x}_{2}+1.7592\right)\left(3 \bar{x}_{1}-3.6388 \bar{x}_{2}-15 \bar{x}_{2}^{2}\right)\right] \mid
\end{aligned}
$$

Then:

$$
\begin{aligned}
v=-\phi(x) \eta_{\epsilon}\left\{0 . 3 \left[1,362\left(\bar{x}_{2}^{2}+1.7592 \bar{x}_{2}-\bar{x}_{3}\right)\right.\right. & \\
& \left.\left.+20 \bar{x}_{1}+680 \bar{x}_{3}\right] \phi(x)\right\}
\end{aligned}
$$

Pick $\epsilon=0.005$.

The control of the process was simulated using the "true" process, Eq. 21, and the robust controller. It is observed, Figure 3, that the output of the true process is ultimately bounded, as predicted. 


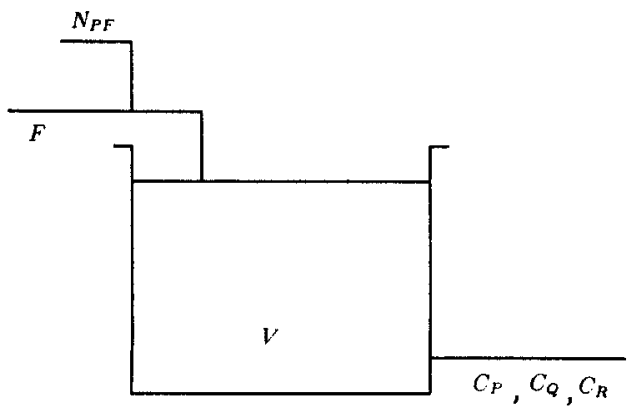

Figure 2. System for example 2.

\section{Example 2}

Consider a CSTR in which an isothermal, liquid-phase, multicomponent chemical reaction is being carried out, Figure 2. The chemical reaction system is:

$$
P \rightarrow Q \rightarrow R
$$
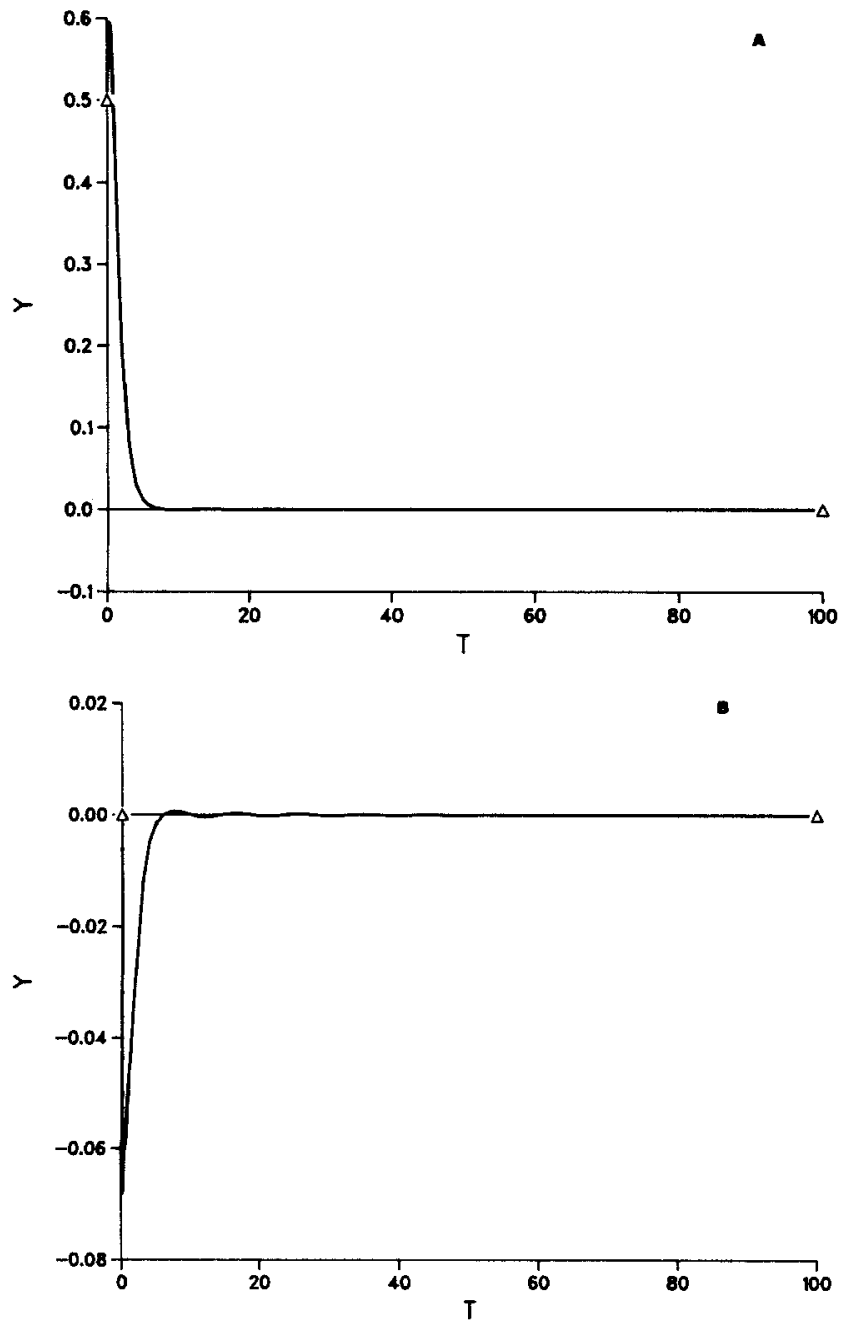

Figure 3. Closed-loop response obtained with robust nonlinear state feedback for example 1.

Figure 3a. Initial conditions: $x_{1}=0.5, x_{2}=0.5, x_{3}=0.5$.

Figure 3b. Initial conditions: $x_{1}=0.6533, x_{2}=-0.5, x_{3}=$ o.o. with the rates of reaction given by:

$$
\begin{aligned}
& r_{1}=k_{5} C_{P} \\
& r_{2}=k_{6} C_{Q}^{2}
\end{aligned}
$$

There is an unmodeled first-order side reaction from $P$. Also there is an error in measuring the molar feed rate of $P . P$ and $Q$ are highly acidic in nature, while $R$ is neutral. To avoid corrosion problems for the downstream equipment the concentration of $P$ and $Q$ must be kept at a given concentration $C_{P 0}$. Thus the control objective is to keep the total concentration of $P$ and $Q$ as close as possible to a concentration $C_{P 0}$ by adjusting the molar feed rate of $P$.

The modeling equations for this system in terms of dimensionless variables are (see the Notation for explanation of symbols):

$$
\begin{aligned}
\frac{d x_{5}}{d t} & =-\left(1+D a_{5}\right) x_{5}+u \\
\frac{d x_{6}}{d t} & =D a_{5} x_{5}-x_{6}-D a_{6} x_{6}^{2} \\
y & =x_{5}+x_{6}
\end{aligned}
$$

where $u=N_{P F} / F C_{P 0}$.

Defining the deviation variables as before we have:

True system

$$
\begin{aligned}
& \dot{x}=[f(x)+\Delta f]+[g(x)+\Delta g] u \\
& y=h(x)+\Delta h
\end{aligned}
$$

Modeled system

$$
\begin{aligned}
& \dot{x}=f(x)+g(x) u \\
& y=h(x)
\end{aligned}
$$

where

$$
\begin{aligned}
f(x) & =\left[\begin{array}{c}
-\left(1+D a_{5}\right) \bar{x}_{5} \\
D a_{5} \bar{x}_{5}-\bar{x}_{6}-D a_{6}\left(\bar{x}_{6}^{2}+2 \bar{x}_{6} x_{6 D}\right)
\end{array}\right] \\
g(x) & =\left[\begin{array}{l}
1 \\
0
\end{array}\right], h(x)=\bar{x}_{5}+\bar{x}_{6} \\
\Delta f & =\left[\begin{array}{c}
-\epsilon_{f 2} \bar{x}_{5} \\
0
\end{array}\right] \text { and } \Delta g=\left[\begin{array}{c}
\epsilon_{g 2} \\
0
\end{array}\right]
\end{aligned}
$$

The values of the various constants are as follows:

$$
\begin{aligned}
D a_{5} & =1.0 \quad D a_{6}=1.0 \quad \epsilon_{f 2}=0.04 \\
\epsilon_{g 2} & =0.25 \quad u_{D}=4.0
\end{aligned}
$$

The steady state values of the states are:

$$
x_{S D}=2.0 \quad x_{6 D}=1.0
$$


To Develop a Robust Controller Based on the Modeled System.

1. Calculation of relative order.

It can easily be verified that $\langle d h, g\rangle \neq 0$ and thus the relative order of the system is 1 .

2. Calculation of state feedback control law:

$$
\bar{u}=\frac{v-\beta_{0}\left(\bar{x}_{5}+\bar{x}_{6}\right)-\beta_{1}\left(f_{1}+f_{2}\right)}{\beta_{1}}
$$

where

$$
\begin{aligned}
& f_{1}=-\left(1+D a_{5}\right) \bar{x}_{5} \\
& f_{2}=D a_{5} \bar{x}_{5}-\bar{x}_{6}-D a_{6}\left(\bar{x}_{6}^{2}+2 \bar{x}_{6} x_{6 D}\right)
\end{aligned}
$$

Making this substitution in the modeled system we have

$$
\begin{aligned}
& \frac{d \bar{x}_{5}}{d t}=\frac{v}{\beta_{1}}-\frac{\beta_{0}}{\beta_{1}}\left(\bar{x}_{5}+\bar{x}_{6}\right)-D a_{5} \bar{x}_{5}-\bar{x}_{6}-D a_{6}\left(\bar{x}_{6}^{2}+2 \bar{x}_{6} x_{6 D}\right) \\
& \frac{d \bar{x}_{6}}{d t}=D a_{5} \bar{x}_{5}-\bar{x}_{6}-D a_{6}\left(\bar{x}_{6}^{2}+2 \bar{x}_{6} x_{6 D}\right)
\end{aligned}
$$

We obtain the "unforced system" by putting $v=0$. It is obvious that the origin is an equilibrium point of the unforced system.

Pick $\beta_{0}=1.0, \beta_{1}=1.0$.

3. Lyapunov function for the unforced system.

Remark 4. We could not find a Liapunov function analytically for the unforced nominal system, so this function was generated numerically using the method of Vannelli and Vidyasagar (1985). The Appendix provides a brief outline of their algorithm; further details may be obtained from their paper.

Using the method of Vannelli and Vidyasagar we computed the following Liapunov function:

$$
V=\frac{R_{2}+R_{3}+R_{4}}{1+Q_{1}+Q_{2}} ; \quad e=6.93 \times 10^{-6}
$$

where

$$
\begin{aligned}
R_{2}= & 0.35007 \bar{x}_{5}^{2}+0.4 \bar{x}_{5} \bar{x}_{6}+0.29985 \bar{x}_{6}^{2} \\
R_{3}= & 0.08366 \bar{x}_{5}^{3}+0.21112 \bar{x}_{5}^{2} \bar{x}_{6}+0.22889 \bar{x}_{5} \bar{x}_{6}^{2}+0.07668 \bar{x}_{6}^{3} \\
R_{4}= & 0.00132 \bar{x}_{5}^{4}+0.01408 \bar{x}_{5}^{3} \bar{x}_{6}+0.02379 \bar{x}_{5}^{2} \bar{x}_{6}^{2} \\
& +0.0144 \bar{x}_{5} \bar{x}_{6}^{3}+0.00245 \bar{x}_{6}^{4} \\
Q_{1}= & 0.2323 \bar{x}_{5}+0.30004 \bar{x}_{6} \\
Q_{2}= & 0.00301 \bar{x}_{5}^{2}+0.02839 \bar{x}_{5} \bar{x}_{6}+0.00177 \bar{x}_{6}^{2}
\end{aligned}
$$

The optimization problem was solved using the subroutine MINOS 5.0.

$V$ has the time derivative:

$$
\dot{V}=-\bar{x}_{5}^{2}-\bar{x}_{6}^{2}
$$

which is negative definite. Thus $V$ is a desirable Lyapunov function.

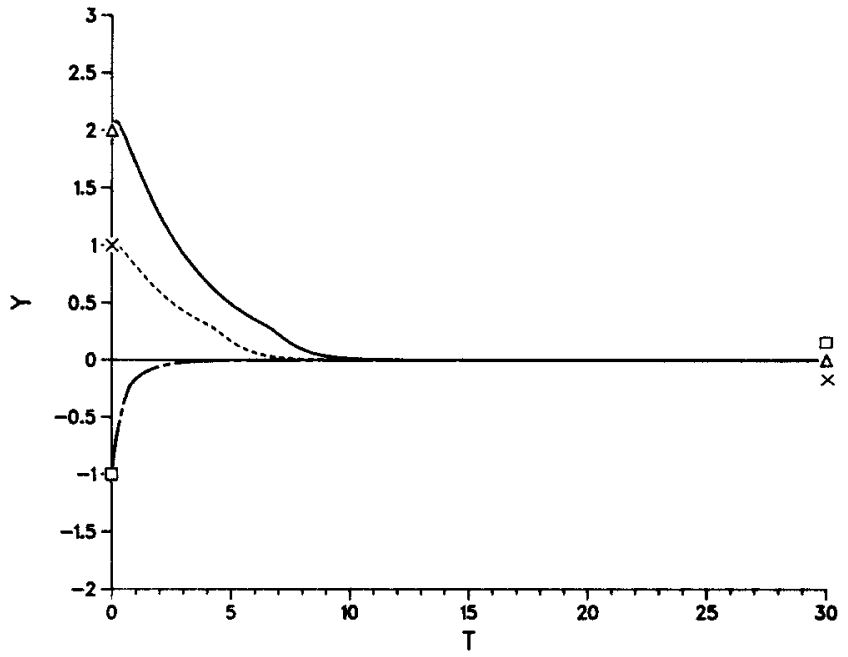

Figure 4. Closed-loop response obtained with robust nonlinear state feedback for example 2. Initial conditions:

$x_{5}=1.0, x_{6}=1.0$

- - $x_{5}=-0.5, x_{6}=-0.5$

$\cdots x_{5}=0.5, x_{6}=0.5$

4. Calculation of $\Delta f^{*}, \Delta g^{*}$ (Eq. 10):

$$
\begin{aligned}
& \Delta f^{*}=-0.04 \bar{x}_{5} \\
& \Delta g^{*}=0.25
\end{aligned}
$$

5. Calculation of $\mu(x), \mu^{0}$ (Eq. 12):

$$
\begin{gathered}
1+\Delta g^{*} \geq \mu(x) \geq 0 \\
\mu^{0} \geq \mu(x)
\end{gathered}
$$

As $\Delta g^{*}=0.25$ set $\mu(x)=\mu^{0}=0.25$.

6. Calculation of $\phi(x)$ (Eq. 13). Set:

$$
\phi(x)=\frac{1}{0.25}\left|-0.04 \bar{x}_{5}-0.25\left(\bar{x}_{6}^{2}+2 \bar{x}_{6}\right)\right|
$$

Then:

$$
v=-\phi(x) \eta_{\epsilon}\left[0.25 \phi(x) \frac{D V}{d \bar{x}_{5}}\right]
$$

Pick $\epsilon=0.003$.

The control of the process was simulated using the "true" process, Eq. 22, and the robust controller. It is observed, Figure 4, that the output of the true process is ultimately bounded, as predicted.

\section{Acknowledgment}

Financial support from the Amoco Foundation is gratefully acknowledged.

The authors are thankful to K. G. Murthy and S. Y. Chang for their help in using the optimization routine MINOS 5.0.

\section{Notation}

$\begin{aligned} C_{A} & =\text { concentration of species } A \text { in reactor, } \mathrm{mol} \cdot \mathrm{m}^{-3} \\ C_{A F} & =\text { feed concentration of species } A, \mathrm{~mol} \cdot \mathrm{m}^{-3} \\ C_{B} & =\text { concentration of species } B \text { in reactor, } \mathrm{mol} \cdot \mathrm{m}^{-3} \\ C_{C} & =\text { concentration of species } C \text { in reactor, } \mathrm{mol} \cdot \mathrm{m}^{-3} \\ C_{P} & =\text { concentration of species } P \text { in reactor, } \mathrm{mol} \cdot \mathrm{m}^{-3}\end{aligned}$ 


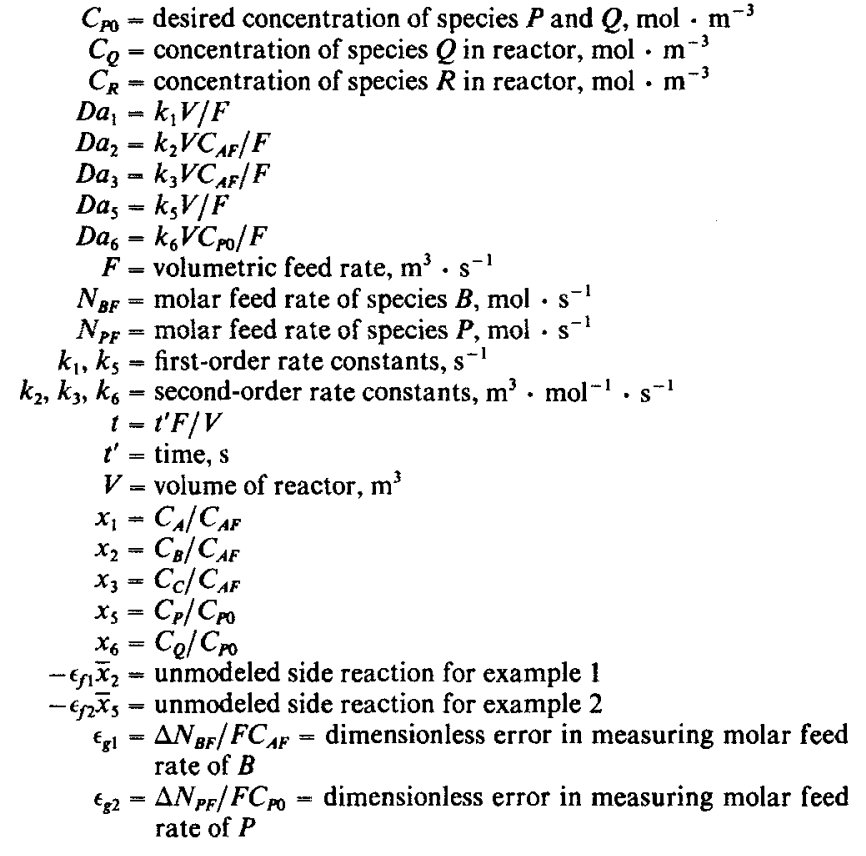

\section{Appendix: Numerical Generation of Liapunov Functions}

The method of Vannelli and Vidyasagar (1985) can be used to generate a Liapunov function numerically. Their algorithm is summarized below.

1. Given

$$
\dot{x}=f[x(t)]
$$

where $f$ is an analytic function and it is known that $x=0$ is an asymptotically stable equilibrium point.

2. As $f$ is analytic, express $f(x)$ as

$$
f(x)=\sum_{i=1}^{\infty} F_{i}(x)
$$

where $F_{i}(\cdot)$ is a homogeneous function of degree $i$. Thus

$$
F_{1}(x)=A x, A \in R^{n x n}
$$

3. Define a Liapunov function candidate $V(x)$ as:

$$
V(x)=\frac{\sum_{i=2}^{n} R_{i}(x)}{1+\sum_{i=1}^{n} Q_{i}(x)}
$$

where $R_{i}$ and $Q_{i}$ are polynomials of degree $i$.

For $V(x)$ to be a Liapunov function, $V(x)$ should be positive definite and $\dot{V}(x)$ should be negative definite over some region. By definition, $\dot{V}(x)$ is negative definite if:

$$
V(x)=[\nabla V(x)]^{\prime} \dot{x}=[\nabla V(x)]^{\prime} f(x)=-x^{\prime} Q x
$$

where $Q$ is positive definite.

4. Find the time derivative of $V(x)$ and equate it to $-x^{\prime} Q x$ and obtain:

$$
\begin{aligned}
& {\left[\left(1+\sum_{i=1}^{n} Q_{i}\right) \sum_{i=2}^{n} \nabla R_{i}-\left(\sum_{i=1}^{n} \nabla Q i\right)\right.}\left.\sum_{i=2}^{n} R_{i}\right] \\
& \cdot \sum_{i=1}^{n} F_{i}=-x^{\prime} Q x\left(1+\sum_{i=1}^{n} Q_{i}\right)^{2}
\end{aligned}
$$

5. Assume a positive definite matrix $Q$ (usually the identity matrix is suitable) and find a positive definite matrix $P$ such that

$$
A^{\prime} P+P A=-Q
$$

Then $V_{2}(x)=R_{2}=x^{\prime} P x$

6. For $n \geq 3$, compare coefficients of like powers of the equation in step 4 . This gives $m$ equations as the coefficients of the polynomials $R_{n}$, and $Q_{n-2}$ are unknown. Find the linear system representation of these $m$ equations; that is find $A y=b$.

Define $e=\|$ coefficients of terms of degree $\geq n+1 \|^{2}$.

7. Solve the following constrained minimization problem:

Min $e$

such that $A y=b$

Let $y^{*}$ be the solution. Use $y^{*}$ to find the coefficients of $R_{n}$ and $Q_{n-2}$. If $e$ is sufficiently small go to step 8; otherwise, increment the value of $n$ by 1 and repeat steps 6 and 7 .

8.

$$
V(x)=\frac{\sum_{1-2}^{n} R_{i}(x)}{1+\sum_{i=1}^{n} Q_{i}(x)}
$$

and

$$
\dot{V}=-x^{\prime} Q x+\frac{(\text { terms of degree } \geq n+1)}{\left(1+\sum_{i=1}^{n-2} Q_{i}\right)^{2}}
$$

If $e=0, \dot{V}=-x^{\prime} Q x$

\section{Literature Cited}

Corless, M. J., and G. Leitmann, "Continuous State Feedback Guaranteeing Uniform Ultimate Boundedness for Uncertain Dynamic Systems," IEEE Trans. Auto. Contr., AC-26, 1139 (1981).

Doyle, J. C., and G. Stein, "Multivariable Feedback Design: Concepts for a Classical/Modern Synthesis," IEEE Trans. Auto. Contr., AC26, 4 (1981).

Gilbert, E., and I. J. Ha, "An Approach to Nonlinear Feedback Control with Applications to Robotics," IEEE Trans. Syst. Man. Cybern., SMC-14, 879 (1984).

Gutman, S., "Uncertain Dynamic Systems--A Liapunov Min-Max Approach," IEEE Trans. Auto. Contr., AC-24, 437 (1979).

Ha, I. J., and E. Gilbert, "Robust Tracking in Nonlinear Systems," IEEE Trans. Auto. Contr., AC-32, 763 (1987).

Hunt, L. R., R. Su, and G. Meyer, "Global Transformations of Nonlinear Systems," IEEE Trans. Auto. Contr., AC-28, 24 (1983a).

_-, "Design for Multi-Input Nonlinear Systems," Differential Geometric Control Theory, R. W. Brockett, R. S. Millman, H. J. Sussman, eds., Birkhauser, Boston, 268 (1983b).

Isidori, A., A. J. Krener, C. Gori-Giorgi, and S. Monaco, “Nonlinear 
Decoupling via Feedback: A Differential Geometric Approach," IEEE Trans. Auto. Contr., AC-26, 331 (1981).

Kantor, J. C., "Stability Constraints for Nonlinear Static State Feedback," Proc. Am. Control Conf.. Minneapolis, 2126 (1987).

Kravaris, C., and C. B. Chung, "Nonlinear State Feedback Synthesis by Global Input/Output Linearization," AIChE J., 33(4), 592 (1987).

Kravaris, C. and S. Palanki, "A Lyapunov Approach for Robust Nonlinear State Feedback Synthesis," IEEE Trans. Auto. Contr., AC-33, in press (1988).

Leitmann, G., "On the Efficacy of Nonlinear Control in Uncertain Linear Systems," ASME J. Dynam. Syst., Meas. Contr., 102, 95 (1981).
Ray, W. H., Advanced Process Control, McGraw-Hill, New York (1981).

Safonov, M. G., Stability and Robustness of Multivariable Feedback Systems, MIT, Cambridge, MA (1980).

Su, R., "On the Linear Equivalents of Nonlinear Systems," Syst. Contr. Lett., 2, 48 (1982).

Vannelli, A., and M. Vidyasagar, "Maximal Lyapunov Functions and Domains of Attraction for Nonlinear Systems," Automatica, 21, 69 (1985).

Manuscript received Oct. 19,1987, and revision received Dec. 22, 1987. 